\title{
The aryl hydrocarbon receptor: a regulator of Th17 and Treg cell development in disease
}

\author{
Peggy P Ho ${ }^{1}$, Lawrence Steinman ${ }^{1}$ \\ ${ }^{1}$ Department of Neurology and Neurological Sciences, Stanford University School of Medicine, Stanford, CA 94305, USA. \\ steinman@stanford.edu \\ Cell Research (2008) 18:605-608. doi: 10.1038/cr.2008.63; published online 2 June 2008
}

The aryl hydrocarbon receptor (AhR) was discovered almost 30 years ago as a specific binding site for the halogenated polycyclic aromatic hydrocarbon, 2,3,7,8-tetrachlorodibenzo-p-dioxin (TCDD), an environmental toxin (as reviewed in [1]). Within the last decade, AhR was found to have a basic helixloop-helix and function as a ligand-activated transcription factor. Located in the cytoplasm of most cells, AhR forms a receptor complex with several proteins including the chaperone protein hsp 90 (a $90 \mathrm{kDa}$ heat shock protein). Once a ligand binds $\mathrm{AhR}$, the ligand-receptor complex translocates to the nucleus and hsp90 is exchanged for the AhR nuclear translocator (Arnt) to form a heterodimer. This heterodimer binds to a DNA recognition site within a gene promoter and activates the expression of several AhR-responsive genes, including prostaglandin synthase $\mathrm{H} 2$ (which converts arachidonic acid into prostaglandins) and the xenobiotic metabolizing enzyme, CYP1A1 (cytochrome P450).

The AhR has a promiscuous ligand binding site, wherein structurally diverse synthetic and naturally occurring AhR ligands have been identified. A review by Denison and Nagy divides AhR ligands into those formed from nonbiological activity (synthetic) and those derived from biological systems as a result of natural processes (natural) [2]. TCDD falls into the synthetic category which includes planar hydropho- bic halogenated aromatic hydrocarbons and polycyclic aromatic hydrocarbons. The natural category includes plantderived materials, such as flavonoids and byproducts derived from converted dietary indoles (i.e., indole 3-carbinol and tryptophan). One such tryptophan photoproduct, 6-formylindolo[3,2b]carbazole (FICZ), is a chemical with significant structural similarity to a high affinity natural AhR ligand, ICZ [2] .

Only recently was TCCD reported to directly induce functional activation of AhR in T cells [3]. Moreover, in 2005, Nohara et al. showed that the activation of AhR can directly inhibit $T$ cell activation and their subsequent proliferation and differentiation, leading to suppression of $\mathrm{T}$ cell help on B cell proliferation [4]. That same year, Negishi and colleagues determined that a synthetic orally active anti-allergic compound, M50367, could also function as an AhR ligand and influence the differentiation of naïve $\mathrm{T}$ cells ( $\mathrm{Th} 0)$ towards T-bet expressing Th1 cells through the downregulation of GATA-3 expression, and thus modulate the Th1/Th2 balance [5]. The question that comes to mind is: are AhR ligands involved in the development of T-cell mediated autoimmune diseases? Two recent articles published in Nature address this hypothesis by studying the role of AhR in the same autoimmune disease model, experimental autoimmune encephalomyelitis (EAE), a CD4+ $\mathrm{T}$ cell mediated animal model for the study of human multiple sclerosis. EAE is induced by the immunization of myelin antigen, such as myelin oligodendrocyte glycoprotein (MOG), combined with adjuvant. The pathogenesis of EAE manifests as an inflammatory process within the central nervous system accompanied by infiltration of the expanded myelin-reactive CD4+ Th1/interleukin 17 (IL-17)-producing Th17 cells and results in repeated attacks on the myelin sheath (demyelination) and nerve damage $[6,7]$.

In the first Nature article, Veldhoen et al. utilized gene array analysis of murine CD4+ effector T-cell subsets to identify the expression of AhR in Th17 cells [8]. Although they found that Arnt was expressed in all CD4+ T cell subsets, AhR was only functional in differentiated Th17 cells. They utilized the tryptophan-derived photoproduct, FICZ, to serve as a high-affinity ligand for AhR. The addition of FICZ during in vitro differentiation of CD4+ effector cells showed no upregulation of AhR or CYP1A1 in Th0, Th1, Th2, or inducible regulatory T cell (Treg) subsets. However, under Th17-cell inducing conditions, the authors observed upregulation of $I L 17 a, I L 17 f$, and IL22 mRNA expression. Retroviral transduction of sorted naïve AhR-deficient CD4+ T cells with an AhR-green fluorescent protein (GFP) showed that only under Th17-cell inducing conditions could IL-22 expression be induced. When Veldhoen and col- 
leagues induced EAE in AhR-deficient mice, they observed delayed onset and less severe disease compared to EAE in wildtype mice, implicating that the lack of functional Th17 cells in AhR-deficient mice dampened disease severity. Co-immunization with FICZ during EAE induction appeared to accelerate disease onset and increase disease severity in wildtype mice, but did not alter the disease in AhR-deficient mice. This again indicated that FICZ binding to AhR was capable of stimulating functionally myelin-reactive Th17 cells. More importantly, the authors found that CD4+ T cells from AhR-deficient mice could still develop into Th17 cells, but they lacked IL-22 expression. Their study concludes that stimulation of AhR by an environmental toxin promotes IL-22 production and enhances Th17 development.

In the second article published in Nature, Quintana and colleagues cloned and characterized the zebrafish homologue of Foxp3 [9]. Zebrafish Foxp3, like its mammalian counterpart, was restricted to lymphocytes. Of note, they identified an evolutionarily conserved binding site for AhR (CABS), and also three non-evolutionary conserved AhR-binding sites (NCABS) in the same region of the Foxp3 promotor where functional binding sites for other transcriptions factors have been found. Chromatin immunoprecipitation analysis of the interaction of AhR with CABS and NCABS in the presence of TCDD showed increased AhR binding, further confirming that AhR controls Foxp3 expression. The authors next utilized Foxp $3^{g f p}$ knockin mice, that have a GFP reporter inserted in the Foxp 3 gene, and showed that purified CD4+ GFP-Foxp3+ Treg cells have increased expression levels of AhR and CYP1A1. Treatment with resveratrol, an AhR antagonist, decreased CYP1A1 and Foxp3 expression, but not AhR expression.

Quintana and colleagues next studied the effect of TCDD on EAE development by administering TCDD one day before EAE induction. They noted that both clinical and histopathological signs of EAE were dramatically reduced. Analysis of day 10 lymph nodes indicated an expansion of Treg cells and decreased frequencies of both Th17 and Th1 cells. Antigenic recall responses of lymph node cells derived from TCDDtreated MOG-immunized mice showed suppressed activation that was reversed in the presence of blocking antibodies to transforming growth factor- $\beta 1$ (TGF- $\beta 1$ ), but not to IL-4 or IL-10. The authors next took $\mathrm{AhR}^{\mathrm{d}}$ mice, which are engineered with a mutation in the AhR ligand binding site leading to reduced affinity for its ligands, and induced EAE. Contrary to the AhR-deficient mice in the study by Veldhoen et al., Quintana and colleagues observed that the $\mathrm{AhR}^{\mathrm{d}}$ mice developed more severe EAE than wildtype mice. Moreover, the $\mathrm{AhR}^{\mathrm{d}}$ mice had increased frequencies of both Th17 and Th1 cells. The authors next took the $\mathrm{AhR}^{\mathrm{d}}$ mice and administered TCDD one day before EAE induction. They found that the $\mathrm{AhR}^{\mathrm{d}}$ mice were readily inducible to EAE disease, and levels of Treg cells did not increase compared to the TCDD treated wildtype mice.

Finally, Quintana and colleagues also confirmed the findings of Veldhoen et al. by showing that AhR expression was highly upregulated in Th17 cells by in vitro activation with either TGF- $\beta 1$ and IL-6, or TGF- $\beta 1$ and IL- 6 with IL23 , or TGF- $\beta 1$ and IL-21 with IL-23. Expression levels of ROR- $\gamma \mathrm{t}$ increased in the presence of FICZ and Th17-cell inducing conditions. FICZ-treated mice were induced for EAE and the result was significant worsening of EAE. These results indicated that FICZ promoted Th17 cell differentiation. Quintana and colleagues conclude that depending on the ligand, AhR is capable of regulating both Treg and Th17 cell differentiation.

From these two Nature articles, there appears to be a dichotomy with the effect of AhR on Th cell differentiation.
First, in the absence of AhR, functional Th17 cell development is hindered by the lack of IL-22 expression [8]. In contrast, when AhR is mutated such that it has lower affinity for its ligand, both Th17 and Th1 cells are expanded and functional [9]. The difference may simply be due to the presence of endogenous $\mathrm{AhR}$ ligands in the $\mathrm{AhR}^{\mathrm{d}}$ mice. Second, it's intriguing that two different high affinity ligands for AhR activation, TCDD and FICZ, can play different roles in the differentiation of Th cells and the pathogenesis of EAE (Figure 1). On the one hand, FICZ, a high affinity "natural" AhR ligand, appears to increase only the Th17 population, and therefore, worsen the severity of EAE [8]. On the other hand, TCDD, a high affinity "synthetic" AhR ligand, appears to expand only the Treg population and effectively prevents EAE [9]. Perhaps the downstream differences are due to the natural evolution of our immune system. If natural food byproducts trigger a food allergy, the immune system would naturally want to control this response by reversing the Th2 response and inducing a Th1/Th17 response. On the other hand, if an environmental toxin were to trigger an unwarranted deleterious immune response, the immune system would in turn want to dampen the effects by inducing a Treg response. Whether this theory holds up, remains to be addressed. Nevertheless, these new findings pave the way for developing AhR-targeted therapies directed towards a multitude of diseases including allergies, cancer, and autoimmune diseases.

CD4+ T-helper (Th) cell differentiation and regulation has been redefined within the last five years by the discovery of IL-17-producing CD4+ Th17 cells (as reviewed in [10]). The original players in the 1980s were the IFN- $\gamma$ and TNF producing Th1 cells and the IL-4 producing Th2 cells. Nearly twenty years later, the Th1/Th2 paradigm had to make way for the IL-10 and TGF- $\beta$ producing Tregs. With Th17 cells as 

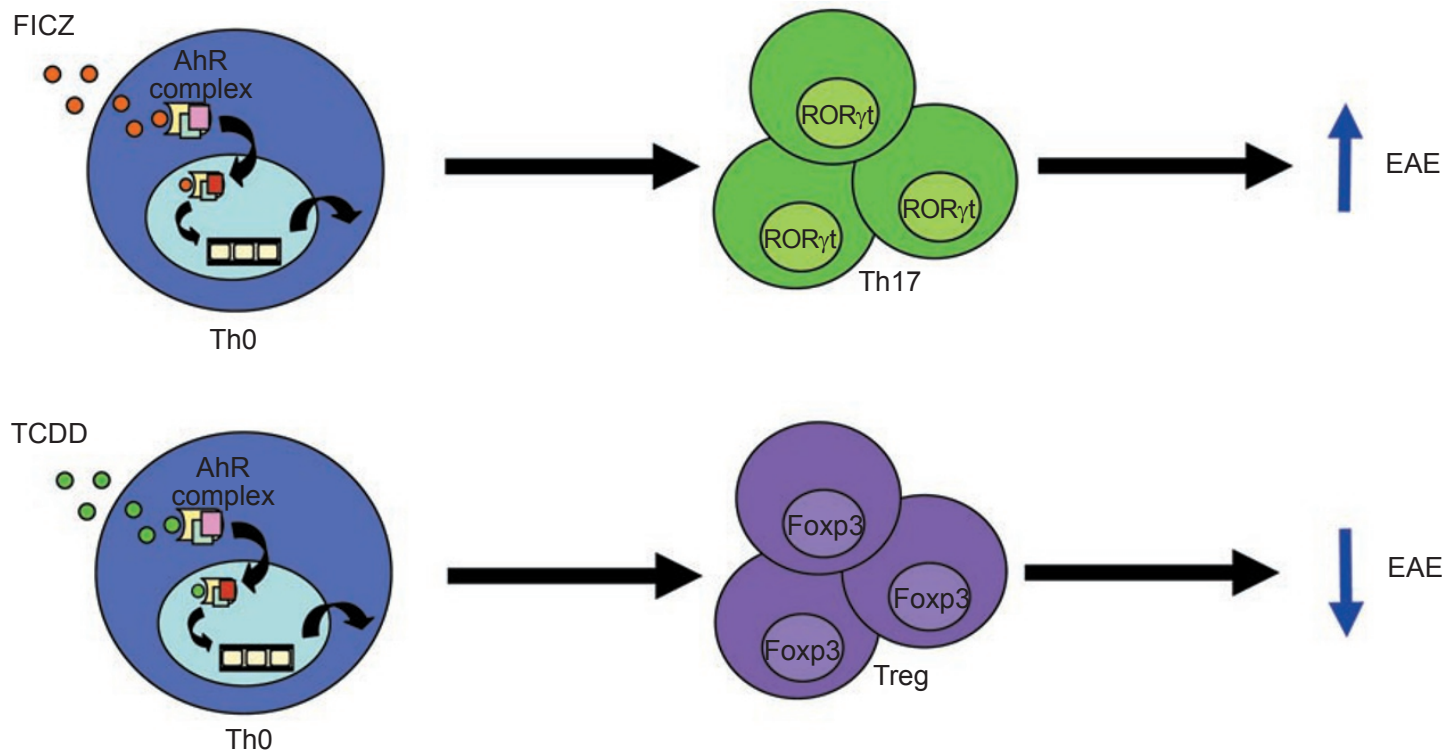

Figure 1 The dichotomy of AhR. FICZ, a high affinity "natural" AhR ligand increases the Th17 population and worsens EAE [8]. TCDD, a high affinity "synthetic" AhR ligand expands the Treg population and prevents EAE [9].

the new kid on the block, a deluge of observations have arisen in an effort to carve out distinctions between the four CD4+ Th cell subsets. The current understanding is that development of Tregs and Th17 are closely related. TGF- $\beta$ alone drives the expression of Foxp3 on T-helper cells and leads to the development of Tregs [6]. In the mouse, naïve Th0 cells stimulated by TGF- $\beta$ and IL- 6 upregulate the expression of orphan nuclear receptor ROR $\gamma \mathrm{t}$ and differentiate into Th17 cells [11]. Recently, McGeachy and colleagues reported that in the mouse TGF- $\beta$ and IL-6 together could drive naïve CD4+ T cells towards Th- 17 commitment, but these Th17 cells would produce both IL-17 and IL-10 and not function as proper effector cells [12]. On the other hand, the additional presence of IL-23 with TGF- $\beta$ and IL- 6 was sufficient to mediate the full pathogenic function of murine Th17 cells [12]. Whether TGF- $\beta$ plays a decisive role in the differentiation of naïve human T cells into Th17 cells is an area of intense controversy at present with published articles showing that TGF- $\beta$ is not critical $[13,14]$.

A recent article in Nature by Zhou et al. [15] confirmed that in the mouse naïve CD4+ T cells receiving a TGF- $\beta$ signal can develop into either a Treg or Th17 cell. Knockdown of Foxp3 expression by using a short hairpin RNA vector, resulted in reduced TGF- $\beta$ induced Foxp3 expression and a partial reversal of TGF- $\beta$-mediated inhibition of ROR $\gamma$ t-directed IL17 expression. Coexpression of Foxp 3 and ROR $\gamma$ t blocked ROR- $\gamma \mathrm{t}$-directed IL-17 expression, and the data suggested that the exon 2-encoded sequence in Foxp3 is required for the interaction between Foxp3 and ROR $\gamma t$. In essence, high concentrations of TGF- $\beta$ induced Foxp 3 which inhibited $I l 23 r$ and ROR $\gamma t$ mRNA expression and suppressed Th17 cell differentiation. Meanwhile, low concentrations of TGF- $\beta$ enhanced $I l 23 r$ mRNA expression which promoted Th17 cell differentiation. Thus, at least in the mouse, TGF- $\beta$-induced Foxp3 is capable of inhibiting ROR $\gamma t$ function in a concentration-dependent manner; however, the addition of IL-6, IL-21, and IL-23 can remove Foxp3-mediated suppression on IL-17 induction and CD4+ T cells can develop into Th17 cells.

\section{References}

1 Okey AB. An aryl hydrocarbon receptor odyssey to the shores of toxicology: the Deichmann Lecture, International Congress of Toxicology-XI. Toxicol Sci 2007; 98:5-38.

2 Denison MS, Nagy SR. Activation of the aryl hydrocarbon receptor by structurally diverse exogenous and endogenous chemicals. Annu Rev Pharmacol Toxicol 2003; 43:309-334.

3 Kerkvliet NI, Shepherd DM, BaecherSteppan L. T lymphocytes are direct, aryl hydrocarbon receptor (AhR)-dependent targets of 2,3,7,8-tetrachlorodibenzo-pdioxin (TCDD): AhR expression in both CD4+ and CD8 $+\mathrm{T}$ cells is necessary for full suppression of a cytotoxic $\mathrm{T}$ lymphocyte response by TCDD. Toxicol Appl Pharmacol 2002; 185:146-152.

4 Nohara K, Pan X, Tsukumo S, et al. Constitutively active aryl hydrocarbon receptor expressed specifically in T-lineage cells causes thymus involution and suppresses the immunization-induced increase in splenocytes. J Immunol 2005; 174:2770-2777.

5 Negishi T, Kato Y, Ooneda O, et al. Effects of aryl hydrocarbon receptor signaling on the modulation of $\mathrm{TH} 1 / \mathrm{TH} 2$ balance. J Immunol 2005; 175:73487356.

6 Bettelli E, Carrier Y, Gao W, et al. Re- 
ciprocal developmental pathways for the generation of pathogenic effector TH17 and regulatory T cells. Nature 2006; 441: 235-238.

7 Komiyama Y, Nakae S, Matsuki T, et al. IL-17 plays an important role in the development of experimental autoimmune encephalomyelitis. J Immunol 2006; 177:566-573.

8 Veldhoen M, Hirota K, Westendorf AM, et al. The aryl hydrocarbon receptor links TH17-cell-mediated autoimmunity to environmental toxins. Nature 2008; 453:106-109

9 Quintana FJ, Basso AS, Iglesias AH, et al. Control of $\mathrm{T}(\mathrm{reg})$ and $\mathrm{T}(\mathrm{H}) 17$ cell differentiation by the aryl hydrocarbon receptor. Nature 2008; 453:65-71.

10 Steinman L. A brief history of $\mathrm{T}(\mathrm{H}) 17$, the first major revision in the $\mathrm{T}(\mathrm{H}) 1 /$ $\mathrm{T}(\mathrm{H}) 2$ hypothesis of $\mathrm{T}$ cell-mediated tissue damage. Nat Med 2007; 13:139145.

11 Ivanov II, McKenzie BS, Zhou L, et al. The orphan nuclear receptor RORgammat directs the differentiation program of proinflammatory IL-17+ T helper cells. Cell 2006; 126:1121-1133.

12 McGeachy MJ, Bak-Jensen KS, Chen Y, et al. TGF-beta and IL-6 drive the production of IL-17 and IL-10 by T cells and restrain $\mathrm{T}(\mathrm{H})-17$ cell-mediated pathology. Nat Immunol 2007; 8:13901397.
13 Wilson NJ, Boniface K, Chan JR, et al. Development, cytokine profile and function of human interleukin 17-producing helper T cells. Nat Immunol 2007; 8: 950-957.

14 Acosta-Rodriguez EV, Napolitani G, Lanzavecchia A, Sallusto F. Interleukins 1 beta and 6 but not transforming growth factor-beta are essential for the differentiation of interleukin 17-producing human T helper cells. Nat Immunol 2007; 8:942-949.

15 Zhou L, Lopes JE, Chong MM, et al. TGF-beta-induced Foxp3 inhibits $\mathrm{T}(\mathrm{H}) 17$ cell differentiation by antagonizing RORgammat function. Nature 2008 Mar 26; doi:10.1038/nature06878. 